\title{
Fatal hemoptysis in patients with advanced esophageal cancer treated with apatinib
}

This article was published in the following Dove Press journal:

OncoTargets and Therapy

\author{
Wei Wang \\ Lin Zhang \\ Yan Xie \\ Tianchang Zhen \\ Gongzhang Su \\ Qi Zang
}

Department of Thoracic Surgery, The Affiliated Qianfoshan Hospital of Shandong University, Jinan, China
Correspondence: Wei Wang

Department of Thoracic Surgery,

The Affiliated Qianfoshan Hospital of

Shandong University, No 16766, Jingshi

Road, Jinan 250014, Shandong, China

Tel +8653I 89268681

Email wangtn8025@sohu.com

\begin{abstract}
Targeted therapy is commonly used for treating advanced malignant tumors. Compared with cytotoxic drugs, targeted drugs have the characteristics of good curative results, less adverse effects, and convenient oral administration. Hence, they are especially suitable for patients with cancer who are not able to tolerate chemotherapy. Anti-angiogenic therapy can achieve the objective by inhibiting the formation of new blood vessels in tumors. Apatinib is a novel tyrosine kinase inhibitor targeting the intracellular domain of vascular endothelial growth factor receptor-2. It has been proven to be effective and safe in treating patients with gastric carcinoma and gastroesophageal junction carcinoma. So far, no reports are available on the treatment of esophageal cancer with apatinib. Two patients with advanced esophageal cancer were treated with oral apatinib because of their poor physical condition. After treatment, the dyspnea symptoms disappeared and quality of life significantly improved. Chest computed tomography showed massive necrosis of tumor tissues in each patient. The tumors significantly reduced and a cavity was formed locally in each patient. However, both patients died of massive hemoptysis, probably due to the rupture of the bronchial artery eroded by tumors. The results indicated that apatinib was effective in treating some patients with advanced esophageal cancer, and adverse effects were controllable. However, doctors should choose appropriate candidates according to apatinib's indications. In addition, the use of apatinib should be carefully controlled for patients with esophageal cancer, especially in those with large vessels and trachea or bronchus eroded by tumor, so as to avoid or reduce the occurrence of fatal hemorrhage.
\end{abstract}

Keywords: angiogenesis inhibitor, esophageal carcinoma, targeted drug, hemoptysis, VEGFR-2

\section{Introduction}

Esophageal cancer is one of the most common malignant tumors of the digestive tract. In China, the number of patients dying of esophageal cancer every year is more than half of the total deaths due to esophageal cancer in the world. ${ }^{1}$ Surgery is still the first choice for treating esophageal carcinoma, but its long-term result is not satisfactory. Postoperative local recurrence and lymph node metastasis are the main reasons for the failure of surgical treatment. ${ }^{2}$ Radiotherapy and chemotherapy are the main treatment methods for patients with advanced esophageal cancer or postoperative recurrence and metastasis. However, no effective treatment is available for patients with poor physical conditions who cannot tolerate chemotherapy.

With the development of molecular biology, targeted therapy has become one important means for treating many malignant tumors, such as lung cancer, breast cancer, and others, due to its good curative result, less adverse effects, and convenient oral administration. ${ }^{3}$ However, no effective targeted drug has been developed for esophageal cancer to date. 
Apatinib is a novel, oral, small molecule, anti-angiogenic agent, which has shown good survival benefits for gastric cancer and esophagogastric junction adenocarcinoma in Phase III clinical trials. ${ }^{4-7}$ However, the efficacy of treating esophageal cancer with apatinib is not clear. Some patients with advanced esophageal cancer were treated using oral apatinib (Hengrui Pharmaceutical Co., Ltd, Jiangsu, China). Of these patients, two achieved significant efficacy, including tumor tissues with massive necrosis. The symptoms improved significantly, but unfortunately, the patients died due to hemoptysis.

\section{Case reports \\ Patient I}

A 51-year-old male was admitted first to the hospital on October 27, 2015, due to "dysphagia for 2 months." Gastroscopy revealed a space-occupying lesion in the middle thoracic esophagus, leading to luminal stenosis. The gastroscope (diameter $6 \mathrm{~mm}$ ) could not pass through the narrow segment. Pathological diagnosis using endoscopic biopsy specimens confirmed that the tumor was a squamous cell carcinoma. A computed tomography (CT) scan revealed a thickened esophageal wall, but there were no enlarged lymph nodes in the mediastinum and distant metastasis (Figure 1). On October 30, 2015, palliative resection of esophageal carcinoma and mediastinal lymph node dissection was performed under general anesthesia. The postoperative pathological findings confirmed that tumor was a moderately differentiated squamous cell carcinoma of the esophagus with a volume of about $6 \times 4 \times 1.5 \mathrm{~cm}^{3}$, penetrating through the outer membrane and involving local peripheral resection surfaces. One positive lymph node was found around the cardia, and the

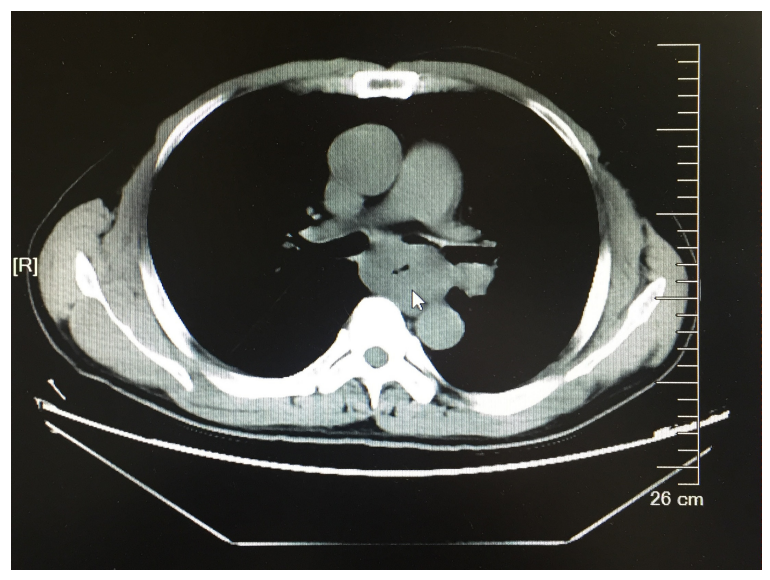

Figure I Preoperative CT image taken in October 2015 showing the presence of a thickened esophageal wall and narrowed esophageal cavity (white arrow). Abbreviation: CT, computed tomography.

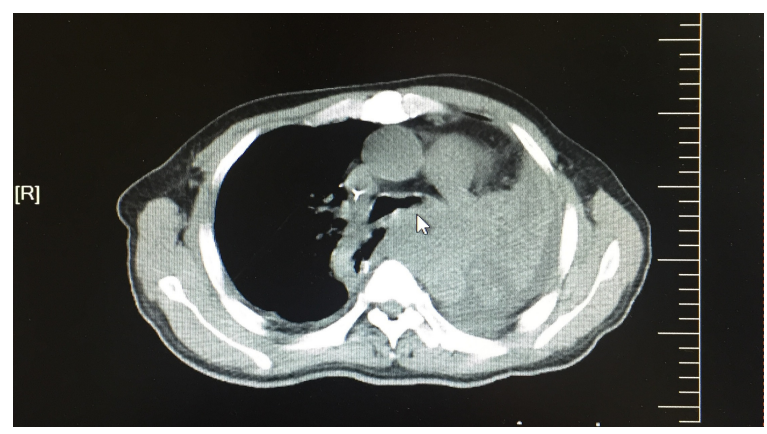

Figure 2 CT image taken in April 2016 showing that after the recurrence of esophageal cancer, the tumor compressed the left main bronchus (white arrow). Abbreviation: CT, computed tomography.

other 14 were negative. On November 30, 2015, the patient accepted radiotherapy with a total dose of $54 \mathrm{~Gy} / 28$ times and synchronized chemotherapy with cisplatin (40 mg, days 1-3) and capecitabine (1,600 $\mathrm{mg}$, days 1 and 8$)$ for four cycles. On April 8, 2016, the patient underwent chest CT examination for chest tightness and cough. The result showed that the tumor recurred, and the left main bronchus was obviously compressed and narrowed (Figure 2). The therapeutic drugs were then replaced with nedaplatin (120 mg, day 1) and docetaxel (60 mg, days 1 and 8) as second-line chemotherapy. After two cycles, the patient's symptoms of chest tightness and cough were relieved, and the CT examination showed an obvious decrease in the volume of the tumor (Figure 3). The left bronchus was unobstructed. Continuing the secondline chemotherapy until the end of the third cycle (October 2016), dyspnea gradually appeared, and the patient could not lie on his back at night. Chest $\mathrm{CT}$ found that the tumor on the mediastinum enlarged again and compressed the left main bronchus (Figure 4). Considering the poor general condition of the patient (performance status score 3) who was unable to

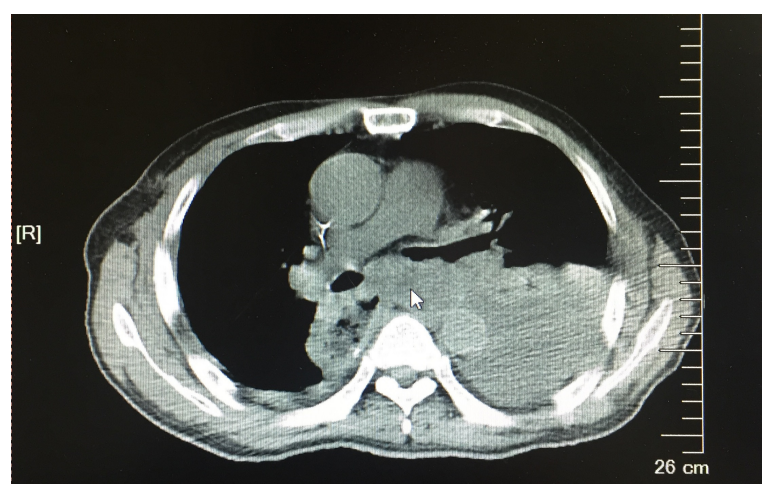

Figure 3 After second-line chemotherapy, chest CT image taken in June 2016 showing that the recurrent tumor had shrunk and the compression on the left main bronchus was alleviated (white arrow).

Abbreviation: CT, computed tomography. 


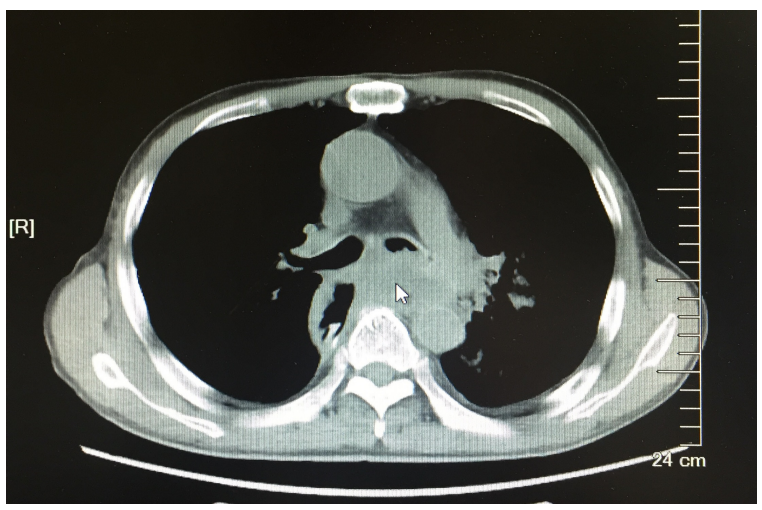

Figure 4 The tumor progressed again and constricted the left main bronchus (white arrow).

Note: The image was taken in August 2016.

tolerate third-line chemotherapy, treatment with oral apatinib $(500 \mathrm{mg} / \mathrm{d})$ was accepted. After 1 week, dyspnea and cough disappeared, and the patient was able to sleep flat at night. Two weeks later, chest CT indicated that the tumor on the mediastinum was obviously necrotic, and a local cavity was formed (Figure 5). The left main bronchial membrane was destroyed with no obvious sign of pulmonary infection. Five weeks later, chest CT found that tumor necrosis was more obvious, the local cavity was further enlarged, and the signs of lung infection were still not apparent (Figure 6). In view of the obvious tumor necrosis, bronchial artery rupture and bleeding were possible. Therefore, the dose of apatinib was changed to $250 \mathrm{mg} / \mathrm{d}$. Unfortunately, the patient died of massive hemoptysis after 7 days. The patient had taken apatinib orally for 47 days. During this period, routine urine and clotting time were monitored regularly. The results showed that the urine protein level fluctuated between probable positive to positive, and the highest activated partial thromboplastin time (APTT) was $37.1 \mathrm{~s}$ (normal 22.7-31.8 s). No hypertension and hand-foot syndrome were found.

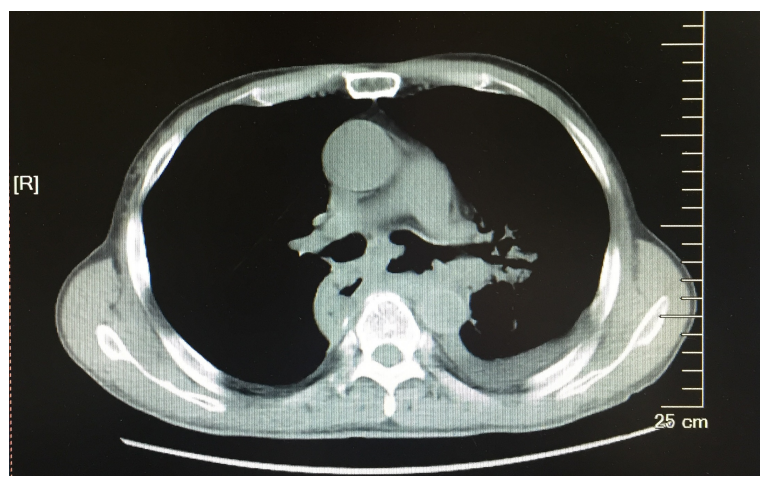

Figure $\mathbf{5}$ The tumor necrosis was obvious, and the local cavity was formed after 2 weeks of oral apatinib.

Note: The image was taken on September I, 2016.

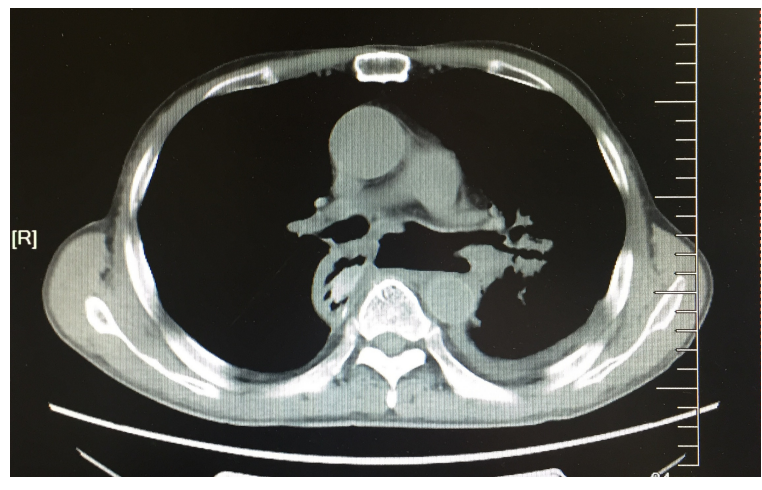

Figure 6 The tumor was further necrotic, and the cavity enlarged after 40 days of oral apatinib.

Note: The image was taken on September 25, 2016.

\section{Patient 2}

A 52-year-old male complained of "dysphagia for 5 months". Gastroscopy showed a neoplasm in the middle thoracic esophagus. The surface of the mass was uneven, hyperemic, and erosive, and ulceration was formed. The pathologic biopsy result was squamous cell carcinoma. The chest CT examination indicated the obvious thickening of the middle thoracic esophageal wall and multiple enlarged lymph nodes in the mediastinum (Figure 7). On November 4, 2016, the patient accepted chemotherapy with cisplatin $(40 \mathrm{mg}$, days 1-3) and docetaxel (120 mg, day 1) for one cycle. Five days after chemotherapy, the patient suddenly felt a sharp, tearing pain in the right chest, associated with fever, and the highest body temperature was up to $40^{\circ} \mathrm{C}$. The thoracic $\mathrm{CT}$ indicated inflammatory changes in the lower lobe of the right lung and homolateral pleural hydropneumothorax. Iodine oil imaging of the upper gastrointestinal tract showed a fistula opening in the anterior right wall of the middle thoracic esophagus. The contrast agent slowly leaked into the thorax

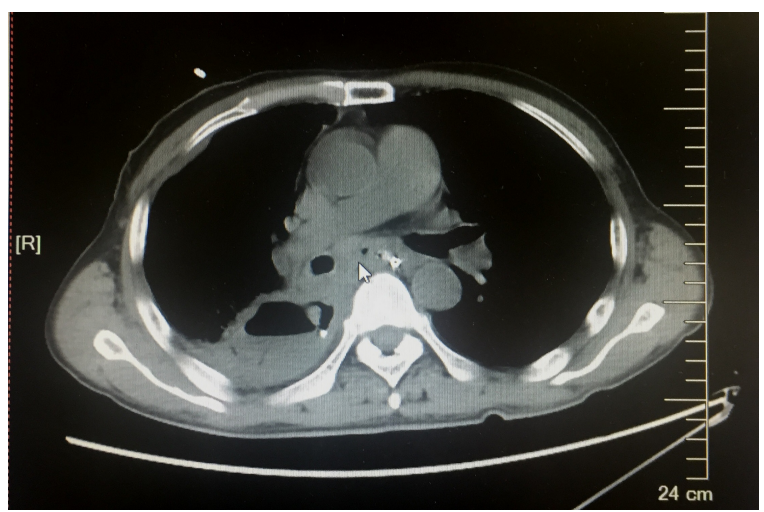

Figure 7 Chest CT image taken on December 16, 2016, showing the presence of a thickened esophageal wall (white arrow).

Abbreviation: CT, computed tomography. 


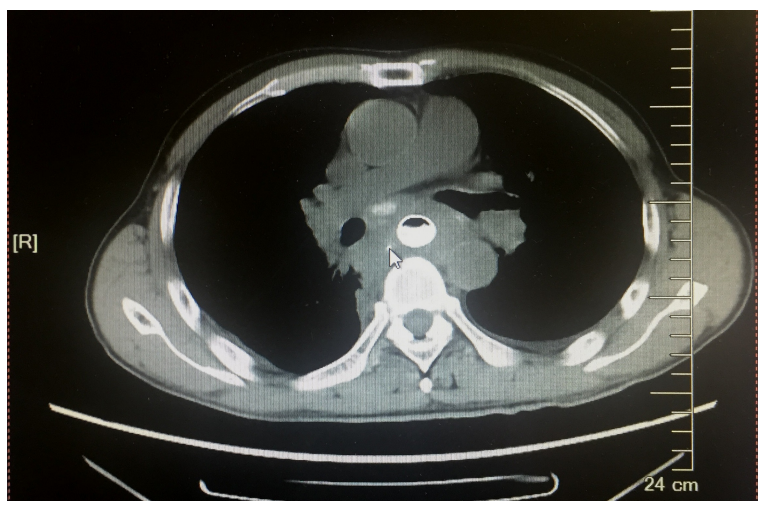

Figure 8 CT image taken on June 12, 20I7, showing that esophageal tumor was significantly larger than before (white arrow).

Abbreviation: CT, computed tomography.

through the opening, confirming the occurrence of esophageal fistula due to the rupture of esophageal cancer. On that day, the patient was admitted for the insertion of a drainage tube into the thoracic cavity and placement of a duodenal nutrition tube to maintain nutrient intake. On November 26 , 2016, an esophageal covered stent was placed into the esophagus through gastroscopy to block the fistula, and the patient was started on a liquid diet after $24 \mathrm{~h}$. Considering the poor general condition of the patient (performance status score 3 ), who was unable to tolerate intravenous chemotherapy, treatment with oral fluorouracil ( $75 \mathrm{mg} /$ twice daily) was started for four cycles, 21 days per cycle. During the treatment, the patient was in a stable condition, the diet was basically normal, and his weight was up about $4.5 \mathrm{~kg}$. On June 12, 2017 , the patient was admitted again for progressive dyspnea. At the same time, his daily actions were severely limited. CT examination results showed that the esophageal tumor was significantly larger than before (Figure 8). On June 16, 2017, the patient began to take apatinib $500 \mathrm{mg} / \mathrm{d}$ orally. Three days later, the symptom of respiratory distress significantly improved. Two weeks later, the chest CT revealed that the esophageal tumor was significantly necrotic, and a local cavity was formed (Figure 9). Subsequently, apatinib was discontinued. On July 4, 2017, a small amount of hemoptysis occurred in the patient, which was stopped using hemostatic drugs. After 4 days, the patient died of massive hemoptysis. The patient had taken apatinib orally for 15 days. During these days, the clotting time examination was normal after 1 week of taking apatinib orally, but 2 weeks later, his plasma prothrombin time (PT $19.9 \mathrm{~s}$, normal 9.8-12.1 s) and APTT (54.20 s) were significantly prolonged. Meanwhile, the other adverse effects such as proteinuria, hypertension, and handfoot syndrome did not occur.

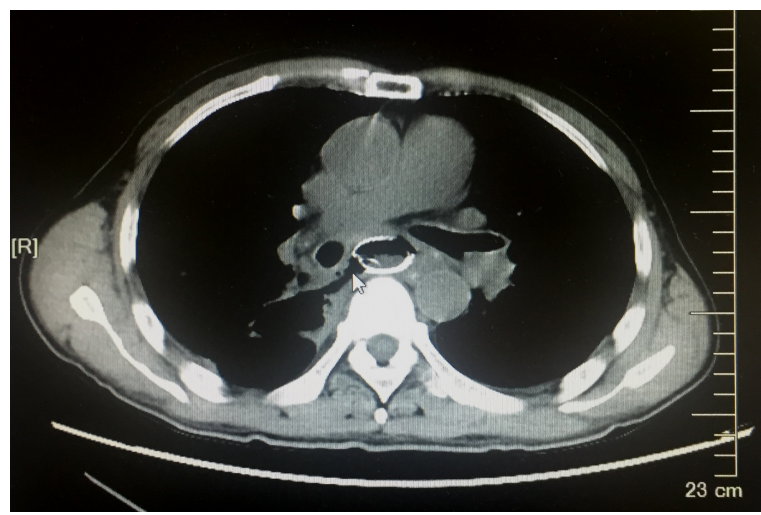

Figure 9 After 2 weeks of treatment with oral apatinib, chest CT image taken on June 29, 2017, showing that the esophageal tumor was significantly necrotic and the local cavity was formed (white arrow)

Abbreviation: CT, computed tomography.

\section{Discussion}

Surgery is still the preferred treatment for patients with esophageal cancer, but more than $40 \%$ of patients have a local or regional recurrence after surgery. ${ }^{8}$ No unified standard treatment exists for these patients; radiotherapy and chemotherapy are the main treatment methods. Although some older patients or patients with poor physical conditions often cannot accept clinical chemotherapy because of drug toxicity, a more safe and effective treatment method is still urgently needed. The molecular targeted drugs have advantages of less adverse effects, convenient administration, and good tolerance compared with cytotoxic drugs. Therefore, they are more accessible to patients with poor physical conditions.

Ferrara and Adamis ${ }^{9}$ believed that tumor angiogenesis could not only provide nutrition for tumors but also act as a pathway for tumor transmission, helping malignant cells escape from primary tumors. Consequently, angiogenesis was vital in neoplastic processes. Therefore, anti-angiogenic drugs have become an important means for the molecular targeted treatment of cancer. Regulation of angiogenesis requires the participation of a variety of signaling molecules. As a major mediating agent for endothelial cell division, survival, and microvascular permeability regulation, vascular endothelial growth factor (VEGF) receptor 2 (VEGFR-2) together with VEGF is crucial in physiological and pathological angiogenesis. ${ }^{10}$ Wang et al ${ }^{11}$ reported that VEGF and VEGFR were highly expressed in squamous cell carcinoma of the esophagus, influencing its development and progression. Therefore, VEGFR might serve as an important therapeutic target for esophageal cancer.

Apatinib is a novel, small molecule anti-angiogenic agent, which can selectively bind to and inhibit VEGFR-2, thus 
inhibiting tumor angiogenesis and growth. ${ }^{12,13}$ Although the use of apatinib in gastric cancer and esophagogastric junction adenocarcinoma showed a good survival benefit in Phase III clinical trials, ${ }^{12,13}$ no study has reported the successful use of this targeted drug in treating patients with advanced esophageal cancer.

The two patients were in the advanced stage of esophageal cancer. They could not tolerate chemotherapy due to the progression of the tumor and the deterioration of the body condition and therefore accepted treatment with oral apatinib. The symptoms of dyspnea in both patients were significantly relieved in a short time after taking the drugs, and their quality of life improved greatly. At the same time, their diseases were controlled for a certain time. Thoracic CT showed massive necrosis of tumor tissues, the tumor volume was significantly reduced, and the compression of surrounding tissues caused by tumors was relieved. These results demonstrated that apatinib was really effective for treating some patients with esophageal cancer. During the treatment, two patients showed an abnormal coagulation function and did not have other complications such as hypertension, proteinuria, and hand-foot syndrome. APTT of patient 1 was slightly prolonged after 3 weeks of treatment using apatinib orally, but patient 2 showed a significant increase in PT and APTT after 2 weeks of treatment. Meanwhile, no obvious abnormality was found in liver and kidney function except for the low plasma protein. This suggested that the abnormal blood coagulation function should be associated with the side effects of apatinib. Therefore, it is believed that patients have a good tolerance for the treatment using apatinib orally as long as their adverse reactions are strictly monitored and controlled in time. Unfortunately, both patients eventually died of massive hemoptysis. The patients' CT images before their death indicated that the tumor continued to progress and invaded the bronchial artery. Further, the treatment with apatinib caused tumor necrosis, leading to hemoptysis by rupture of vessels.

Although only two patients with advanced esophageal cancer were treated effectively using apatinib in this study, the findings provide some valuable insights. 1) Apatinib has an obvious effect on some patients with advanced esophageal cancer and can improve their symptoms in a short time. The imageological examination showed obvious tumor necrosis and cavity formation, but the tissues surrounding the tumor were also damaged. Therefore, not only a regular chest CT examination is necessary but also the follow-up interval should be shortened. The medication should be adjusted in time for patients with obvious tumor necrosis and cavity formation. 2) Hemorrhage or abnormal clotting time is one of the main side effects of anti-angiogenic drugs, and it often occurs within 1 month after taking the drugs. Therefore, the blood clotting function should be monitored regularly. The patients with hematemesis, hemoptysis, or black stool should reduce or stop the medicine. 3) Physicians should focus on choosing suitable candidates. For those with middle and upper thoracic esophageal cancer, especially with large vessels, trachea or bronchus eroded by tumor, or esophageal tracheal fistula, apatinib should be used with caution to reduce the risk of fatal bleeding.

\section{Conclusion}

Apatinib can serve as an ideal treatment option through strictly mastering the indications, closely monitoring the toxic side effects, and adjusting drug solutions in time for patients with advanced esophageal cancer who have poor physical conditions and are unable to tolerate chemotherapy.

\section{Acknowledgments}

The authors thank editors at MedSci and Ms Jingwen Liu for English language copyediting.

The authors confirm they have obtained written informed consent of the patients' next of kin for publication of these case reports and the related images.

\section{Disclosure}

The authors report no conflicts of interest in this work.

\section{References}

1. Jemal A, Bray F, Center MM, Ferlay J, Ward E, Forman D. Global cancer statistics. CA Cancer J Clin. 2011;61(2):69-90.

2. Wang YX, Yang Q, He M, et al. Patterns of recurrence in patients with stage III thoracic esophageal squamous cell carcinoma after radical resection. Zhonghua Zhong Liu Za Zhi. 2017;39(1):48-55.

3. Jin Z, Yoon HH. Antiangiogenic therapy in gastroesophageal cancer. Hematol Oncol Clin North Am. 2017;31(3):499-510.

4. Qin SK, Li J. Experts consensus on the clinical application of apatinib in gastric cancer treatment. Chin Clin Oncol. 2015;20(9):841-847.

5. Li J, Qin S, Xu J, et al. Randomized, double-blind, placebo-controlled phase III trial of apatinib in patients with chemotherapy-refractory advanced or metastatic adenocarcinoma of the stomach or gastroesophageal junction. J Clin Oncol. 2016;34(13):1448-1454.

6. Chen LT, Oh DY, Ryu MH, et al. Anti-angiogenic therapy in patients with advanced gastric and gastroesophageal junction cancer: a systematic review. Cancer Res Treat. 2017;49(4):851-868.

7. Huang L, Wei Y, Shen S, et al. Therapeutic effect of apatinib on overall survival is mediated by prolonged progression-free survival in advanced gastric cancer patients. Oncotarget. 2017;8(17):29346-29354.

8. Li B, Zhang WC, Zhao LJ, et al. Therapeutic strategy to prevent the recurrence of esophageal carcinoma after radical resection. Chin Clin Oncol. 2013;40(24):1553-1557. 
9. Ferrara N, Adamis AP. Ten years of anti-vascular endothelial growth factor therapy. Nat Rev Drug Discov. 2016;15(6):385-403.

10. Hwang J. Beyond HER2: recent advances and future directions in targeted therapies in esophagogastric cancers. J Gastrointest Oncol. 2016; 7(5):763-770.

11. Wang H, Zhiyin F, Xiao C. Expression and significance of VEGFR2 in esophageal squamous cell carcinoma of Kazaks. J Xinjiang Med Univ. 2016;39(9):1197-1200.
12. Ding J, Chen X, Dai X, Zhong D. Simultaneous determination of apatinib and its four major metabolites in human plasma using liquid chromatography-tandem mass spectrometry and its application to a pharmacokinetic study. JChromatogr B Analyt Technol Biomed Life Sci. 2012;895(7):108-115.

13. Zhang H. Apatinib for molecular targeted therapy in tumor. Drug Des Devel Ther. 2015;9(11):6075-6081.

\section{Publish your work in this journal}

OncoTargets and Therapy is an international, peer-reviewed, open access journal focusing on the pathological basis of all cancers, potential targets for therapy and treatment protocols employed to improve the management of cancer patients. The journal also focuses on the impact of management programs and new therapeutic agents and protocols on

patient perspectives such as quality of life, adherence and satisfaction. The manuscript management system is completely online and includes a very quick and fair peer-review system, which is all easy to use. Visit http://www.dovepress.com/testimonials.php to read real quotes from published authors. 